MS37 Molecular compounds

and MOFs at ambient

conditions and under high

pressure

Chairs: Francesca Fabbiani, Wendy Queen

MS37-01 The Effect of Pressure, Guest

Uptake and Structural Flexibility on Porous Framework Materials

Stephen Moggach ${ }^{1}$

1. University of Edinburgh, Scotland

email: s.moggach@ed.ac.uk

In recent years the development of new methods of storing, trapping or separating light gases, such as $\mathrm{CO}_{2}$, $\mathrm{CH}_{4}$ and $\mathrm{CO}$ has become of outmost importance from an environmental and energetic viewpoint. Porous materials such as zeolites and porous organic polymers have long been considered good candidates for this purpose. More recently, metal organic frameworks (MOFs) have attracted further interest with many aspects of their functional and mechanical properties investigated. The porous channels found in MOFs are ideal for the uptake of guests of different shapes and sizes, and with careful design they can show high selectivity for particular species from a mixture. Adsorption properties of MOFs have been thoroughly studied, however obtaining in depth 'structural' insight into the adsorption/desorption mechanism is not so common place. For example, out of ca. forty thousand published framework structures there are less than 10 entries in which $\mathrm{CO}_{2}$ molecules have been unambiguously located experimentally within the pores.

Over the last 6 years, we have been using high-pressure crystallographic techniques to explore the uptake of guest species in the pores of MOFs. We do this, by taking advantage of the fact that the small molecules that encompass the pressure transmitting fluids used frequently in high-pressure crystallographic studies, can penetrate the pores on increasing pressure. This has revealed unexpected flexibility, explain unusual adsorption phenomena under milder gas pressures, and increase reactivity in MOFs. The potential for using high-pressure to explain structure-property relationships has also been revealed in porous magnetic materials. Here, we will give an overview of the effect of high-pressure on both micro and nanoporous materials, and in-particular, highlight some recent work on gas-loaded framework materials.

\section{MS37-02 Role of pressure transmitting media in structural transformations of molecular crystals at high pressures}

Boris Zakharov $^{1,2}$, Sergey V. Goryainov ${ }^{3}$, Yurii V. Seryotkin ${ }^{1,2,3}$, Nikolay A. Tumanov ${ }^{4}$, Elena V. Boldyreva ${ }^{1}$

1. Institute of Solid State Chemistry and Mechanochemistry SB RAS (Novosibirsk, Russia)

2. Novosibirsk State University (Novosibirsk, Russia)

3. V.S. Sobolev Institute of Geology and Mineralogy (Novosibirsk, Russia)

4. Institute of Condensed Matter and Nanosciences, Université catholique de Louvain (Louvain-la-Neuve, Belgium)

email: b.zakharov@yahoo.com

Molecular compounds are interesting both from fundamental and applied point of view since many of them are promising as materials with piezoelectric, ferroelectric and non-linear optical properties. Some of them can serve as biomimetics. Due to these facts studies of polymorphism of molecular compounds are important for crystal engineering and crystallization theory. High pressure is one of the most powerful tools to influence polymorphism of molecular compounds. However, the control of high-pressure polymorphism is not a trivial task. Generally, it is not possible to predict which phase will be formed at a selected $(\mathrm{T}, \mathrm{P})$ point based on a thermodynamic phase diagram. As a result of the kinetic control of nucleation and nuclei growth, different phases can form, depending on the choice of the starting polymorph, the hydrostatic medium, the compression/decompression protocol and the choice of pressure transmitting media. The last effect is the most intriguing due to the fact that proper pressure transmitting media do not react chemically with studied molecular compound. Despite that pressure transmitting medium was shown to influence the product of high-pressure transformation of molecular compounds. In this contribution we illustrate this phenomenon in relation to an antidiabetic drug, chlorpropamide. In addition to the commercially available $\alpha$ polymorph, which is thermodynamically stable, four other polymorphs $(\beta-, \gamma-$, $\delta-, \varepsilon-)$ can be preserved indefinitely long under ambient conditions. The variety of polymorphs makes chlorpropamide very interesting for high-pressure research. The chlorpropamide polymorphs were studied by diffraction and spectroscopic techniques. We compared the phase transitions in solid polymorphs immersed in different pressure transmitting media. We also studied the effects of pressure, when several different polymorphs were present simultaneously in the same diamond anvil cell. A plethora of interesting effects was observed. The same polymorph of chlorpropamide was shown to undergo transformations into different polymorphs depending on selected pressure transmitting medium (without recrystallization), as well as to recrystalize into other forms, depending on the presence of other polymorphs in the same diamond anvil cell.

The work was supported by a grant from RSF (14-13-00834).

Keywords: structural transformations, high pressures, polymorphism 